\title{
Targeting PSD95/nNOS by ZL006 alleviates social isolation-induced heightened attack behavior in mice
}

\author{
Luqi Yang ${ }^{1} \cdot$ Jingyu Cui ${ }^{1} \cdot$ Ligong Zeng $^{1} \cdot$ Wen Lu $^{2,3}$ (])
}

Received: 19 July 2021 / Accepted: 10 October 2021 / Published online: 18 October 2021

(c) The Author(s), under exclusive licence to Springer-Verlag GmbH Germany, part of Springer Nature 2021

\begin{abstract}
Rationale Deregulated attack behaviors have devastating social consequences; however, satisfactory clinical management for the behavior is still an unmet need so far. Social isolation (SI) has been common during the COVID-19 pandemic and may have detrimental effects on mental health, including eliciting heightened attack behavior.

Objectives This study aims to explore whether injection of ZL006 can alleviate SI-induced escalation of attack behavior in mice.

Methods Pharmacological tools, biochemical methods, and behavioral tests were used to explore the potential therapeutic effects of ZL006 targeting postsynaptic density 95 (PSD95)/neuronal nitric oxide synthase (nNOS) pathway on escalation of attack behavior induced by SI in mice.

Results ZL006 mitigated SI-induced escalated attack behaviors and elevated nitric oxide (NO) level in the cortex of the SI mice. The beneficial effects of ZL006 lasted for at least $72 \mathrm{~h}$ after a single injection of ZL006. Potentiation of NO levels by L-arginine blocked the effects of ZL006. Moreover, a sub-effective dose of 7-NI in combination with a sub-effective dose of ZL006 decreased both SI-induced escalated attack behaviors and NO levels in mice subjected to SI.

Conclusions Our study highlights the importance of the PSD95/nNOS pathway in mediating SI-induced escalation of attack behavior. ZL006 may be a promising therapeutic strategy for treating aggressive behaviors.
\end{abstract}

Keywords Postsynaptic density 95 (PSD95) · Neuronal nitric oxide synthase (nNOS) · ZL006 · Nitric oxide (NO) · Social isolation (SI) · Resident-intruder test

\section{Introduction}

The neuronal mechanisms underlying aggressive behavior, particularly deregulated attack behaviors, have been studied extensively. However, clinical management of this disorder remains unsatisfactory. Social isolation (SI), which has been common during the COVID-19 pandemic, poses detrimental effects on human mental health and probably increases the

Wen Lu

swkxlw@163.com

1 Department of Clinical Medicine, Hainan Medical University, Haikou, China

2 Department of Biochemistry and Molecular Biology, School of Basic Medicine and Life Sciences, Hainan Medical University, Haikou, Hainan, China

3 Key Laboratory of Molecular Biology, School of Basic Medicine and Life Sciences, Hainan Medical University, Haikou, Hainan, China risk of aggressive behaviors (Brooks et al. 2020; Cacioppo et al. 2015; Cacioppo et al. 2014; Killgore et al. 2021; Okruszek et al. 2020; Peterson et al. 2021). SI plays a critical role in the etiology of aggressive behavior and is known to provoke an escalation of attack behavior in mice (Pinna et al. 2003; Zelikowsky et al. 2018).

Glutamatergic signaling, acting via the N-methyl-Daspartate receptor (NMDAR) and its downstream effector, nitric oxide (NO), is involved in a large number of psychiatric disorders (Deyama et al. 2017; Zhou et al. 2007; Zhu et al. 2014). Preclinical studies have demonstrated that these disorders can be alleviated by inhibiting hyperactivity of NMDAR or aberrant production of NO initiated by activated NMDAR (Baranyi et al. 2015; Dhir and Kulkarni 2011; Doucet et al. 2013; Tripathi et al. 2020; Zhou and Sheng 2013). However, blockade of NMDAR via its antagonists is undermined by psychotomimetic side effects (Muir 2006; Olney et al. 1991) possibly due to NMDAR playing numerous crucial functions under physiology conditions. 
Scaffolding protein postsynaptic density 95 (PSD95) tethers NMDAR to neuronal nitric oxide synthase (nNOS), which is responsible for the synthesis of $\mathrm{NO}$ after activation of NMDAR (Sattler et al. 1999). Although several lines of evidence suggest that nNOS and perhaps other types of NOS are involved in aggressive behavior, findings regarding the roles of NOS are conflicting based on previous studies (Carreno Gutierrez et al. 2020; Demas et al. 1999; Nelson et al. 1995; O'Leary et al. 2020).

4-(3,5-Dichloro-2-hydroxy-benzylamino)-2-hydroxybenzoic acid (ZL006) is a small molecule that targets PSD95/ nNOS and inhibits NMDAR-dependent NO synthesis in neurons with an IC50 of dozens of nanomoles and can cross the blood-brain barrier after systemic administration (Zhou et al. 2010). Selective targeting of PSD95/nNOS, but not NMDAR or NO, circumvents the side effects caused by global inhibition of the receptor and NO. Therefore, ZL006 exhibits potential to serve as a therapeutic agent for the treatment of escalated attack behaviors.

In this study, we investigated whether intraperitoneal (i.p.) injection or intracerebroventricular (i.c.v.) injection of ZL006 can alleviate SI-induced heightened attack behaviors in mice.

\section{Materials and methods}

\section{Subjects}

Male C57BL/6J mice (aged 3-4 or 7-8 weeks) were purchased from SILAC (Shanghai SLAC Laboratory Animal CO. Ltd, Shanghai, China). The animals were maintained on a reversed $12 \mathrm{~h} / 12 \mathrm{~h}$ light/dark cycle with food and water ad libitum. All surgical procedures and treatments were approved by the Experimental Animal Ethics Committee of Hainan Medical University (No. XPT170075) and performed in accordance with the NIH Animal Research Use guidelines for the Care and Use of Laboratory Animals. Maximal efforts were made to minimize the number of mice used on the experiments and any suffering.

\section{Drugs}

4-(3,5-Dichloro-2-hydroxy-benzylamino)-2-hydroxybenzoic acid (ZL006, T17293; TargetMol, tsbiochem, Shanghai, China) was administered via i.p. injection at a dose of $2 \mathrm{mg} /$ $\mathrm{kg}$ or $10 \mathrm{mg} / \mathrm{kg}$ or via i.c.v injection at a dose of $10 \mu \mathrm{M} / 2.5$ $\mu \mathrm{L}$ or $50 \mu \mathrm{M} / 2.5 \mu \mathrm{L}$ in $0.9 \%$ saline. $\mathrm{L}$-arginine $(750 \mathrm{mg} /$ kg, A5006; Sigma-Aldrich, St Louis, MO, USA), or 7-NI (10 mg/kg, T7474; TargetMol, tsbiochem, Shanghai, China) was injected via the i.p. route. All drugs were freshly prepared on the day of use and were injected as indicated before testing. The doses of ZL006 were selected based on our pilot experiments or reports by other groups (Aziz et al. 2019; Doucet et al. 2013; Lee et al. 2015; Qu et al. 2020).

\section{Intracerebroventricular injection}

Briefly, mice were anesthetized (using sodium pentobarbital $50 \mathrm{mg} / \mathrm{kg}$, via i.p. injection) and positioned in a stereotaxic instrument with a mouse adapter. The stereotaxic coordinates for implantation of a guide cannula into the right lateral ventricle were as follows: $\mathrm{AP}, 0.5 \mathrm{~mm}$ relative to bregma; ML, $0.8 \mathrm{~mm}$ relative to bregma; and DV, -2.5 $\mathrm{mm}$ from the skull surface. The guide cannula was fixed with dental cement. All injections were administered over 1 min, and the syringe was kept in place for 2 min to prevent backflow after injection. To confirm successful entry into the right ventricle before our main experiments, $5 \mu \mathrm{L}$ of trypan blue dye was injected into the cannula, and the mouse brain was cut into slices for observation. All animals were allowed to recover for 2 weeks after the operations before initiation of the subsequent experiments. Control animals received a vehicle solution. During drug infusion, the animals were gently restrained by hand; the stylets were removed from the guide cannula and replaced by 27 -gauge injection needles ( $1 \mathrm{~mm}$ below the tip of the guide cannula). Each injection was connected to a $10-\mu \mathrm{L}$ Hamilton syringe using a polyethylene tube.

\section{Open field test}

The open field test was performed as described previously with minor modifications (Ai et al. 2020; Ai et al. 2017). The open field chamber was made of white plastic with dimensions of $40 \mathrm{~cm}$ height $\times 40 \mathrm{~cm}$ length $\times 40 \mathrm{~cm}$ width. The mice were allowed to explore the chamber freely for $10 \mathrm{~min}$. Ethanol (70\%) was used to clean the apparatus between subjects to eliminate the odor cue. The total distance traveled during the test was measured using Super Maze software (Shanghai Xinruan Information Technology Co. Ltd, Shanghai, China).

\section{Resident-intruder test}

The resident-intruder test was used to measure aggressive behavior. SI mice were housed singly for 2 weeks, and intruder mice were housed at a density of 3-4 mice per cage before the test. The behavior of each resident mouse was scored blindly for total attack count, attack duration, and attack latency. An attack was defined as a single bite or a flurry of rapid bites initiated by the resident. The bedding in the cages housing the resident mice was not renewed for 3 days before the test. 


\section{NO level measurement}

Immediately after the resident-intruder test, the cortex of each mouse was harvested and stored at $-80{ }^{\circ} \mathrm{C}$ until examination. The cortical tissue was lysed using lysis buffer (P0013, Beyotime, Shanghai, China) with protease inhibitors. NO levels were measured using the Griess reaction (Hevel and Marletta 1994; Zhang 2001). The assay was performed according to the manufacturer's instructions (S0021S; Beyotime, Shanghai, China). Absorbance of the samples was measured at $540 \mathrm{~nm}$ using a microplate reader (BioTek Instruments, VT, USA). The nitrite concentration was calculated using a standard curve and expressed as a relative value.

\section{Statistical analysis}

Statistical analyses were conducted in GraphPad Prism 7 (GraphPad Software Inc., CA, USA). Data were scatterplotted in the bar which indicates the mean with an error bar representing the standard error of the mean (SEM). Statistical analysis between two groups was tested by the two-tailed Student's $t$ test. One-way analysis of variance (ANOVA) with Bonferroni's correction was performed for multiple comparisons between each group. "N" indicates animal number; the sample size was predetermined by our previous experience and similar studies. Differences at $p<0.05$ were considered significant. Detailed statistical analysis can be found in the supplemental information.

\section{Results}

\section{ZL006 attenuates SI-induced attack behaviors and aberrant NO levels in the cortex}

The open field and resident-intruder tests were performed consecutively to assess the locomotor activity and attack behaviors in the group housing $(\mathrm{GH})$ and SI mice treated with vehicle or different doses of ZL006 (Fig. 1a). After intraperitoneal administration, it takes about $1 \mathrm{~h}$ for ZL006 to reach a maximal level in the brain (Zhou et al. 2010). Therefore, the behavior tests were conducted $1 \mathrm{~h}$ after administration. As found in a previous study (Doucet et al. 2013), ZL006 had minimal effects on locomotor activity, with no overt between-group differences (Fig. 1b). After 2 weeks of single housing, the SI mice displayed markedly heightened attack behaviors, which manifested as an increase in the number and duration of attacks and a shorter attack latency in comparison with the $\mathrm{GH}$ mice that received a vehicle injection (Fig. 1c-e), indicating that SI induced an escalation in attack behavior. There was no change in attack behavior in the GH mice that received ZL006 (Fig. 1c-e), suggesting that this drug neither nonspecifically decreases attack behavior nor interferes with an animal's ability to protect its territory. Given that ZL006 inhibits PSD95/nNOS and that abnormal amounts of NO in the brain are linked to neurological disorders, including aggressive behavior, we harvested the cortex after behavioral tests and assessed the NO level using the Griess reaction (Hevel and Marletta 1994; Zhang 2001). In line with the behavioral results in this study, there was a marked reduction in NO levels in the SI mice treated with high-dose ZL006 but not in the SI mice treated with vehicle or low-dose ZL006 (Fig. 1f). Another cohort of mice was subjected to the resident-intruder test to determine whether a single ZL006 injection had a sustained effect on attack behaviors (Fig. 1g). Our data indicate that the effect on attack behaviors of one ZL006 injection lasted for at least $72 \mathrm{~h}$ (Fig. 1h-m). The NO level was also reduced $72 \mathrm{~h}$ later in the high-dose ZL006-treated SI mice (Fig. 1n). Overall, our results demonstrate that ZL006 efficiently mitigates SI-induced escalation of attack behavior without interfering with locomotor activity and thus has potential as a therapeutic agent for aggressive behavior.

\section{Intracerebroventricular injection of ZL006 reduced SI-induced attack behaviors and aberrant NO levels}

To determine whether ZL006 had a central rather than a peripheral impact on mouse behavior, we administered ZL006 via the i.c.v route (Tillmann et al. 2017) to determine its effect on SI mice (Fig. 2a). As anticipated, the mice treated with vehicle or ZL006 displayed no impairment in the total distance in the open field test (Fig. 2b). When compared with vehicle-treated or low-dose ZL006-treated (ZL L) mice, high-dose ZL006-treated (ZL H) mice showed a lower attack count, a shorter attack duration, and a longer attack latency, suggesting a direct reduction in aggressive behaviors (Fig. 2c-e). After the behavioral tests, we measured the NO levels in the mouse cortices. As with the intraperitoneal injection of ZL006, the i.c.v injection of high-dose ZL006 attenuated the SI-induced increase in NO levels (Fig. 2f). Overall, these data demonstrate that ZL006 attenuates escalation of attack behavior induced by SI and leads to a concomitant decrease in NO levels in the cortex.

\section{Pretreatment with L-arginine prevented the effects of ZL006 on SI-induced attack behaviors and aberrant NO levels}

L-arginine, a known precursor of production of $\mathrm{NO}$ in vivo (Moncada and Higgs 1993), was used to examine whether elevated levels of $\mathrm{NO}$ are required for aggressive behavior in mice subjected to SI. To address this, we assessed locomotor activity and attack behaviors following administration of L-arginine and ZL006 using the open field test and 

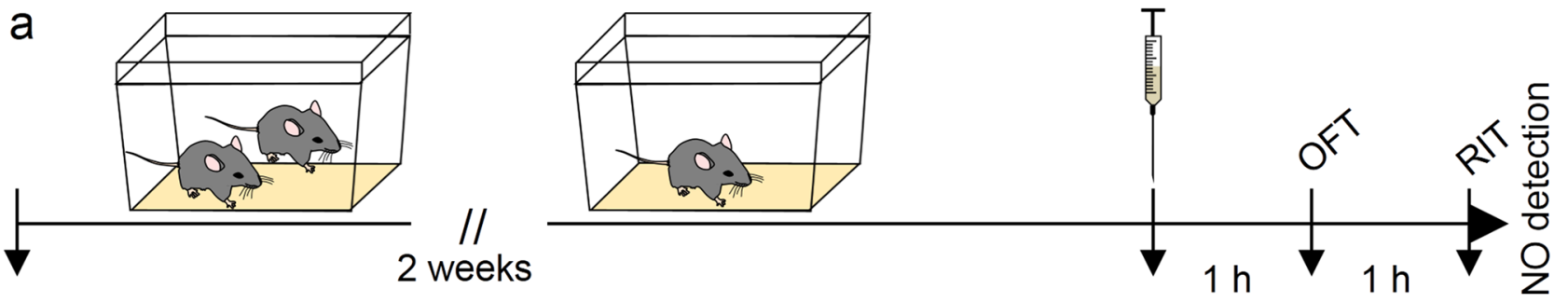

$\mathrm{GH}+$ Veh $\quad \mathrm{GH}+\mathrm{ZL} \mathrm{H} \quad \mathrm{SI}+\mathrm{Veh} \quad \mathrm{SI}+\mathrm{ZLL} \quad \square \mathrm{SI}+\mathrm{ZL} \mathrm{H}$

b c d
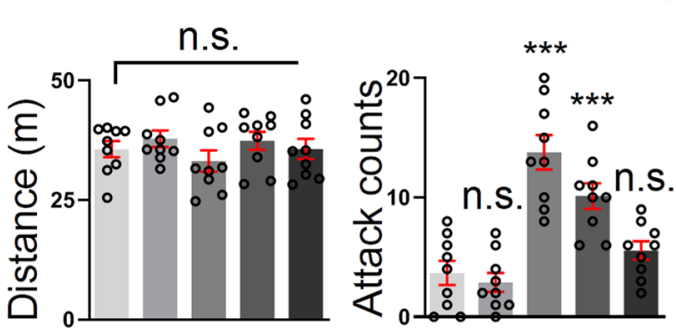

d $\quad e$

e f

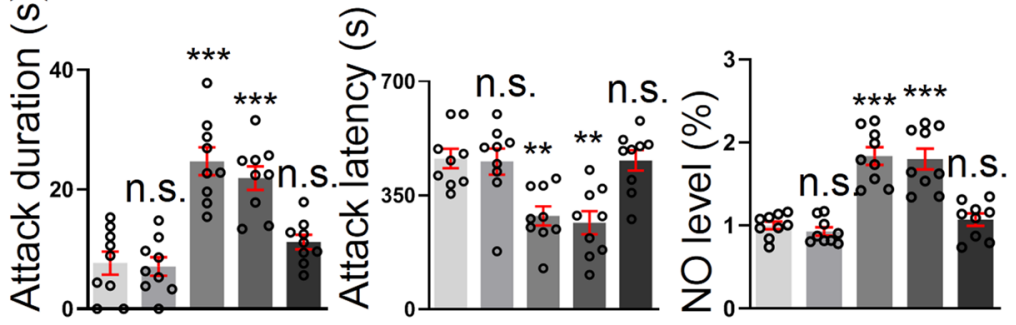

g
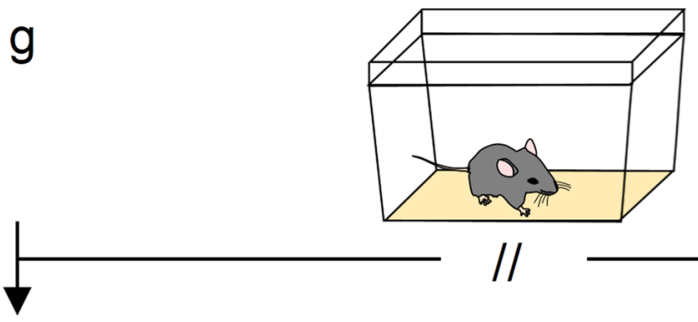

SI+Veh $\quad$ SI+ZL H

$24 \mathrm{~h}$
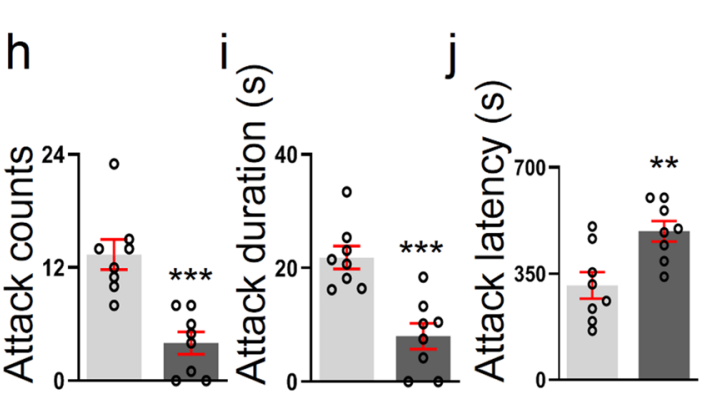

Fig. 1 Systemic injection of ZL006 suppresses SI-evoked escalated attack behaviors and augmented NO level in mice. (a) Diagram of the experimental design. After 2 weeks of group housing (GH) or single housing (social isolation, SI), the mice were treated with vehicle (Veh) or different doses of ZL006 (2 mg/kg for a low dose, indicated by ZL L; $10 \mathrm{mg} / \mathrm{kg}$ for a high dose, denoted by ZL $\mathrm{H})$ via intraperitoneal injection. (b) Total distance of mice during the open field test (OFT) treated as shown in (a) $\left(F_{(4,40)}=0.9094, p=\right.$ 0.4678). (c-e) Parameters of the attack behavior measured during the resident intruder test (RIT). Total attack counts $(\mathbf{c})\left(F_{(4,40)}=19.56, p\right.$ $<0.001)$; attack duration $\left(\right.$ d) $\left(F_{(4,40)}=19.94, p<0.001\right)$; and attack latency (e) $\left(F_{(4,40)}=8.859, p<0.001\right)$. (f) Relative NO level $\left(F_{(4,40)}\right.$ $=27.33, p<0.001)$. (g) Scheme of the protocol used. $(\mathbf{h}-\mathbf{j})$ Attack behavior parameters measured $24 \mathrm{~h}$ after administration of ZL006 ( $10 \mathrm{mg} / \mathrm{kg}$ for a high dose, denoted by ZL H) injection during the resident intruder test. Total attack counts (h) $(t=4.71, p<0.001)$, attack duration in (i) $(t=4.572, p<0.001)$, and attack latency in $(\mathbf{j})$ $(t=3.23, p=0.0061) 24 \mathrm{~h}$ later. $(\mathbf{k}-\mathbf{m})$ Total attack counts $(\mathbf{k})(t=$ 5.758, $p<0.001)$, attack duration (l) $(t=5.62, p<0.001)$, and attack latency $(\mathbf{m})(t=4.492, p<0.001) 72 \mathrm{~h}$ later. (n) Relative NO levels $(t=5.897, p<0.001)$. ** $p<0.01$; *** $p<0.001$; n.s., not statistically significant; all were compared with GH + Veh $(\mathbf{b}-\mathbf{f})$ or SI + Veh (h-n). In all figures, the individual data plots represent each animal within the group overlaying the bar indicating the mean and the error bar indicating the standard error of the mean (SEM). Detailed statistical analysis can be found in the supplemental information 

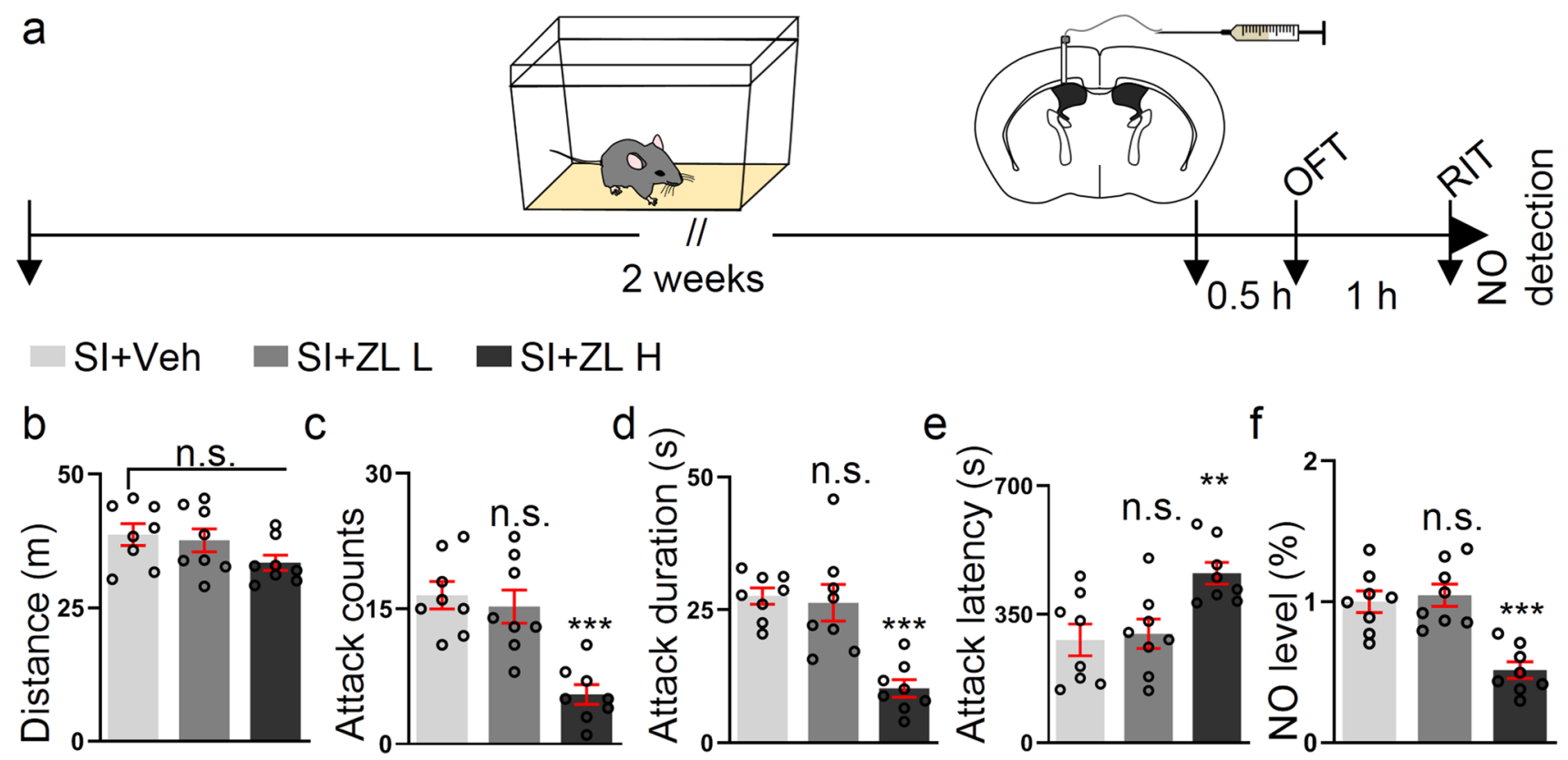

Fig. 2 Intracerebroventricular injection of ZL006 reduces SI-evoked attack behaviors and elevated NO levels in mice. (a) Schematic drawing of the experimental protocol. After 2 weeks of SI, the mice were treated with vehicle (Veh) or different doses of ZL006 $(10 \mu \mathrm{M} / 2.5 \mu \mathrm{L}$ for a low dose, indicated by ZL L; $50 \mu \mathrm{M} / 2.5 \mu \mathrm{L}$ for a high dose, denoted by ZL H) via intracerebroventricular injection. (b) Total distance of mice $\left(F_{(2,21)}=2.119, p=0.1451\right)$. (c-e) Attack behav-

the resident-intruder test, respectively (Fig. 3a). No apparent differences in distance were detected in the open field test in any group (Fig. 3b), suggesting that neither ZL006 nor L-arginine affected locomotor activity. Pretreatment of ZL006-treated mice with the vehicle consistently resulted in a lower attack count, a shorter attack duration, and a longer attack latency, indicating a substantial reduction in aggressive behavior (Fig. $3 \mathrm{c}-\mathrm{e}$ ). However, pretreatment with L-arginine in ZL006-treated mice attenuated the effects of ZL006, as evidenced by comparable attack counts, attack duration, and attack latency relative to the vehicle only group (Fig. 3c-e). Notably, mice that were pretreated with $\mathrm{L}$-arginine in combination with the vehicle did not show an increase in attack behaviors (Fig. 3c-e), suggesting SI-induced ceiling effects on attack behaviors. Indeed, there was a marked reduction in NO levels in mice treated with vehicle + ZL006, and this decrease was reversed by pretreatment with L-arginine (Fig. 3f). However, L-arginine in combination with the vehicle did not boost the NO level further compared with that in the vehicle + vehicle-treated mice (Fig. 3f). Overall, these results suggest that potentiation of NO production by L-arginine blocked the effect of ZL006 on attack behaviors, suggesting that NO plays an essential role in SI-induced attack behaviors. ior parameters measured during the resident intruder test. Total attack counts $(\mathbf{c})\left(F_{(2,21)}=15.73, p<0.001\right)$, attack duration $(\mathbf{d})\left(F_{(2,21)}=\right.$ $16.68, p<0.001)$, and attack latency $(\mathbf{e})\left(F_{(2,21)}=6.971, p=0.0048\right)$. (f) Relative NO level $\left(F_{(2,21)}=16.78, p<0.001\right)$. ** $p<0.01$; *** $p$ $<0.001$; n.s., not statistically significant; all were compared with SI + Veh. Detailed statistical analysis can be found in the supplemental information

\section{Combined treatment with sub-effective doses of ZL006 and 7-NI had a synergistic effect on SI-induced attack behaviors and aberrant NO levels}

To further ascertain the role of ZL006 in targeting PSD95/ nNOS and reducing heightened attack behaviors, we employed a pharmacological approach to simultaneously inhibit PSD95/nNOS and nNOS activity with sub-effective doses of ZL006 and 7-NI, a preferential nNOS inhibitor (Mutlu et al. 2011), and evaluated the effects of this manipulation on attack behaviors using the resident-intruder test (Fig. 4a). There was no difference in total distance between the treatments as measured in the open field test (Fig. 4b). Subsequently, we observed a combined effect of co-application of sub-effective doses of ZL006 and 7-NI, as demonstrated by a marked reduction in the attack count and duration of attacks and a longer attack latency (Fig. 4c-e). However, no significant changes in attack behaviors were detected in mice that received a sub-effective dose of ZL006 or 7-NI alone when compared with mice treated with vehicle only (Fig. 4c-e). Notably, there was a marked decrease in NO levels in mice co-injected with sub-effective doses of ZL006 and 7-NI but not in those that received sub-effective ZL006 or sub-effective 7-NI alone (Fig. 4f). These findings 


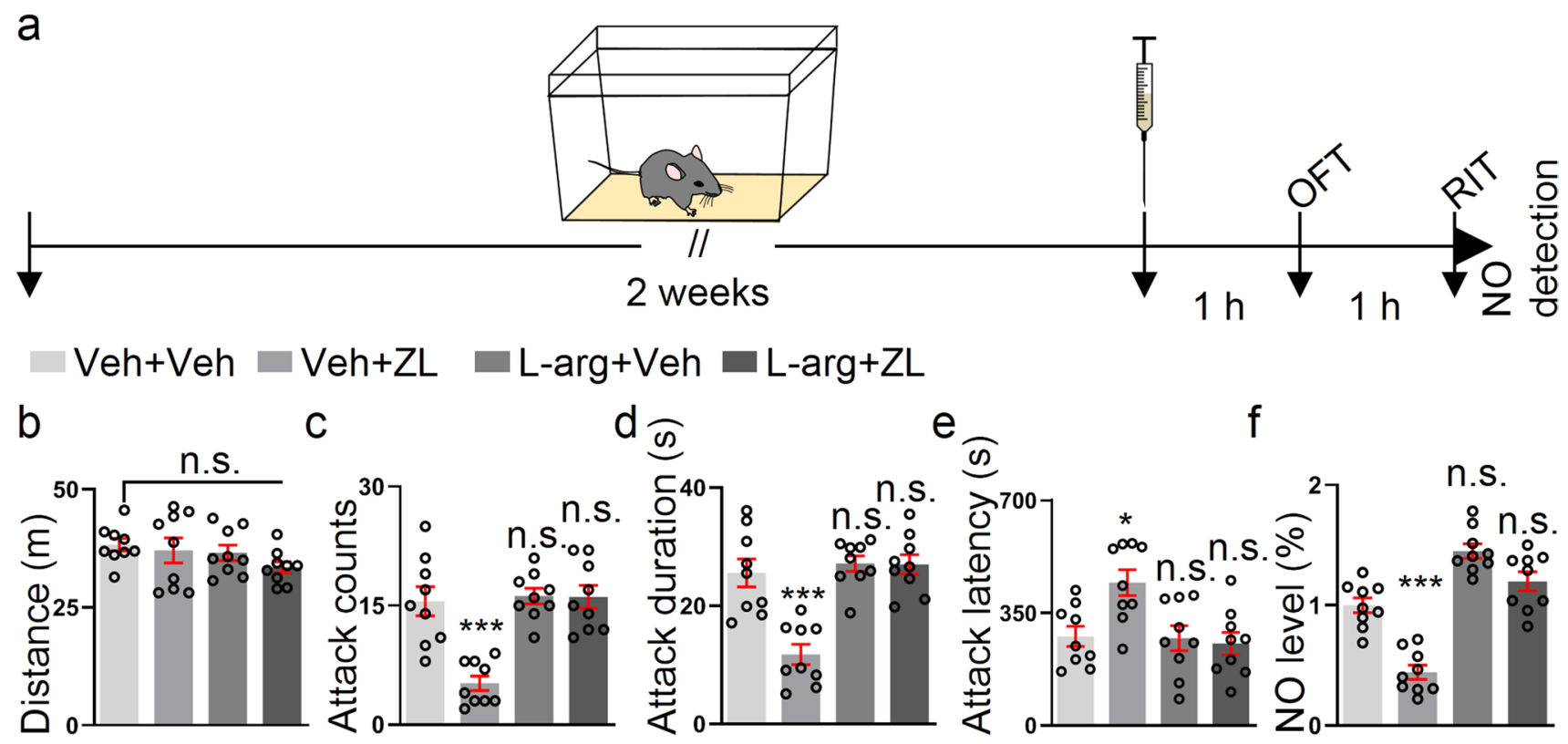

Fig. 3 Pretreatment with L-arginine abolishes the effect of ZL006 on SI-evoked attack behaviors and elevated NO levels in mice. (a) Schematic drawing of the experimental protocol. After 2 weeks of SI, the mice were treated with vehicle (Veh) or L-arginine (L-arg) via intraperitoneal injection followed by vehicle (Veh) or ZL006 (ZL) via intraperitoneal injection 30 min later. (b) Total distance of mice $\left(F_{(3,32)}=1.302, p=0.2908\right)$. $(\mathbf{c}-\mathbf{e})$ Attack behavior parameters

further support our theory that specifically disrupting PSD95/nNOS by ZL006 alleviates SI-induced escalation of attack behavior by reducing elevated NO levels.

\section{Discussion}

Despite its prevalence and disabling consequences, the biological mechanisms underlying deregulated attack behavior remain poorly understood, and there are no effective clinical management strategies. In this study, a single dose of ZL006, a small-molecule inhibitor of PSD95/ nNOS, attenuated escalation of attack behavior in the resident-intruder test at a dose that did not affect locomotor activity. To our knowledge, this is the first study to demonstrate that targeting PSD95/nNOS with ZL006 attenuates attack behavior induced by SI, thereby adding to the evidence for ZL006 as a potential treatment for various neurological disorders (Cai et al. 2018b; Doucet et al. 2013; Li et al. 2018; Qin et al. 2019). Notably, we demonstrated that the effect of ZL006 on attack behavior was mediated via suppression of production of NO. The actions observed in mice treated with ZL006 in our study have paved the way for future dose optimization in preclinical trials and subsequent clinical trials. Besides, we also inspired other studies that employ similar agents targeting the PSD95/ measured in the resident intruder test. Total attack count $(\mathbf{c})\left(F_{(3,32)}=\right.$ $15.89, p<0.001)$, attack duration $(\mathbf{d})\left(F_{(3.32)}=17.07, p<0.001\right)$, and attack latency (e) $\left(F_{(3,32)}=5.876, p=0.0026\right)$. (f) Relative NO level $\left(F_{(3,32)}=32.97, p<0.001\right) . * p<0.05 ; * * p<0.01 ; * * * p<0.001$; n.s., not statistically significant; all were compared with Veh + Veh. Detailed statistical analysis can be found in the supplemental information

nNOS or its downstream node in the pathway, including IC87201 or ZLc002 (Florio et al. 2009; Lee et al. 2018; Lee et al. 2015; Zhu et al. 2014).

NMDAR-mediated production of NO is an important mechanistic pathway in neurological disorders (Carey et al. 2017; Gunasekar et al. 1995; Sattler et al. 1999). Therefore, suppression of NMDAR-mediated aberrant NO levels is a promising strategy for the amelioration of various neurological disorders. However, inhibitors that directly target NMDAR and NOS have a number of side effects, which means that there is an urgent need for alternative approaches to inhibit NMDAR-dependent production of NO. Considering that PSD95 tethers nNOS to NMDAR and that selective disruption of PSD95/nNOS spares memory and motor function (Smith et al. 2016), we hypothesized that ZL006, a validated pharmacological tool in numerous rodent models of neurological disease (Carey et al. 2017; Qu et al. 2020; Sherwin et al. 2017), would suppress the attack behaviors induced by SI. Given previous research demonstrating that ZL006 has a limited ability to penetrate the blood-brain barrier (Wang et al. 2015a), we also administered ZL006 via the i.c.v route to test the central action of the drug. Our data consistently indicated that a single injection of ZL006 can alleviate SI-induced attack behaviors whether administered via the i.p. route or the i.c.v route. Therefore, targeting PSD95/nNOS downstream of the NMDAR may be a more 


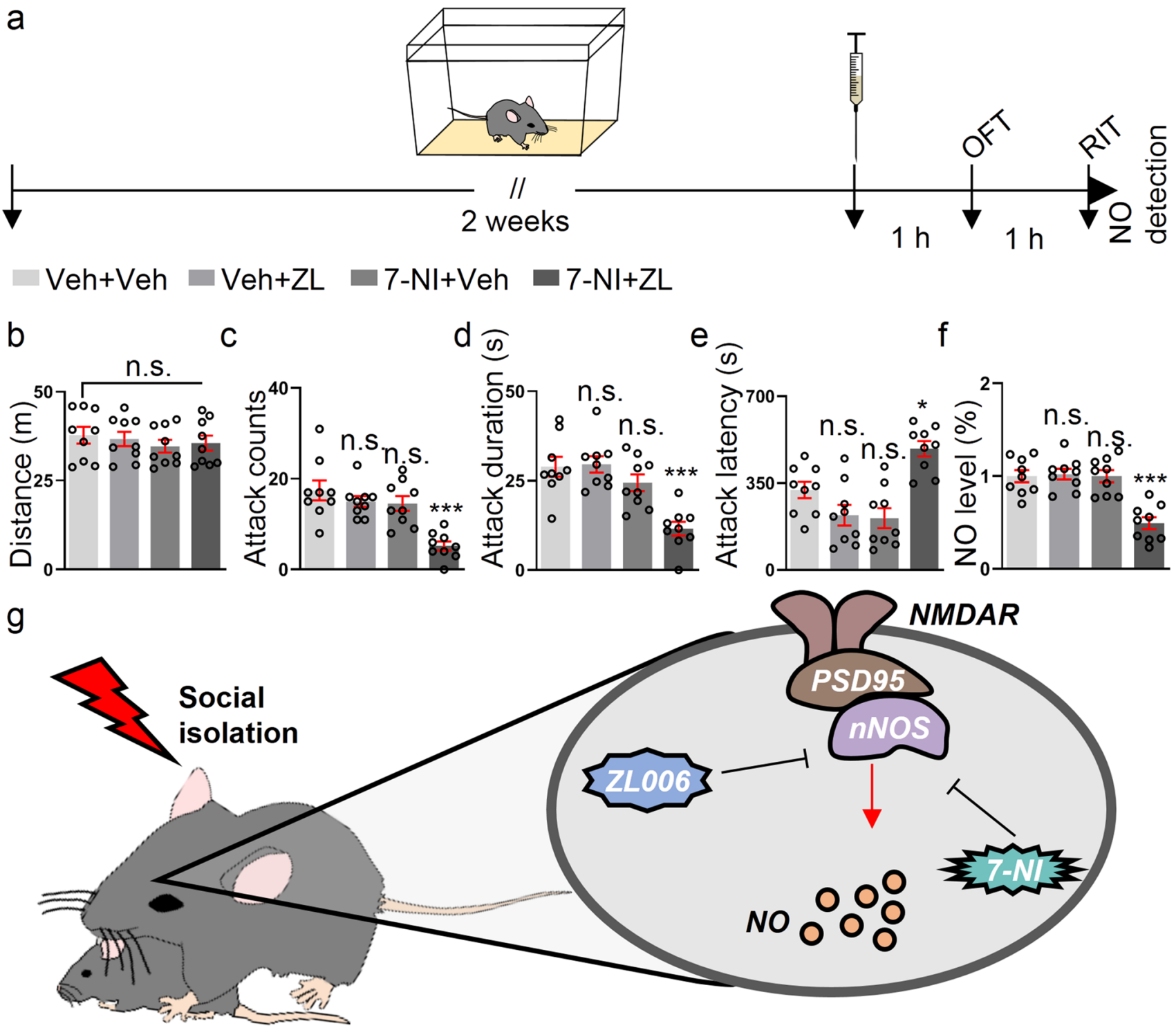

Fig. 4 Sub-effective dose of ZL006 and 7-NI had synergistic effects on SI-evoked attack behaviors and elevated NO levels in mice. (a) Schematic drawing of the experimental protocol. After 2 weeks of SI, the mice were treated with vehicle (Veh) or 7-NI via intraperitoneal injection followed by vehicle (Veh) or ZL006 (ZL) via intraperitoneal injection 30 min later. (b) Total distance of mice $\left(F_{(3,32)}=0.4225, p\right.$ $=0.7381)$. $(\mathbf{c}-\mathbf{e})$ Attack behavior parameters measured during the resident intruder test. Total attack counts $(\mathbf{c})\left(F_{(3,32)}=11.62, p<0.001\right)$, attack duration $(\mathbf{d})\left(F_{(3,32)}=12.61, p<0.001\right)$, and attack latency $(\mathbf{e})$

promising therapeutic strategy for attack behavior than an NMDAR antagonist.

The role of NO in attack behavior is subtle and multifaceted, and the evidence is mixed with regard to whether elevated or reduced NO levels contribute to escalation of attack behavior (Carreno Gutierrez et al. 2020; Demas et al. 1999; Nelson et al. 1995). These seemingly contradictory reports may reflect differences in the strains of mice used in different laboratories (Roy et al. 2000). Another possible
$\left(F_{(3,32)}=12.43, p<0.001\right)$. (f) Relative NO level $\left(F_{(3,32)}=16.55, p<\right.$ 0.001). (g) Summary of findings. Two weeks of SI elicited heightened attack behavior and deregulated the PSD95/nNOS pathway, which caused an elevated NO level, while ZL006 or sub-effective doses of ZL006 and 7-NI reduced the attack behavior and reversed the increment in the NO level. $* p<0.05$; ** $p<0.01 ; * * * p<0.001$; n.s., not statistically significant; all were compared with Veh + Veh. Detailed statistical analysis can be found in the supplemental information

explanation for this paradox is that NO plays biphasic roles in vivo (Getting et al. 1996), suggesting that impaired homeostasis of the NO levels in vivo may result in abnormal attack behaviors. Furthermore, the expression of mRNA of nNOS is upregulated in more aggressive zebrafish (Filby et al. 2010; Freudenberg et al. 2016), and reduction of NO levels in zebrafish was found to suppress aggression (Carreno Gutierrez et al. 2020), implying that the effect of NO is species-specific. In line with the notion that an increase 
in the NO level contributes to escalation of attack behavior (Carreno Gutierrez et al. 2020; Demas et al. 1999), our data demonstrate that restoration of a normal NO level by ZL006 averts SI-induced attack behaviors. However, little is known about the functional role of NO before, during, and after this behavior. This knowledge gap can be bridged by the development of a NO sensor (Eroglu et al. 2018), which can be exploited in an intact system to detect changes in the NO level in situ and in real time. However, further studies are warranted to elucidate the role of NO in attack behaviors.

Intriguingly, the effect of ZL006 was sustained for at least $72 \mathrm{~h}$ following a single injection. Considering that low-dose ZL006 had a minimal impact on attack behaviors and the pharmacokinetics of ZL006 (Wang et al. 2015; Zhou et al. 2010), it is unlikely that a single injection of high-dose ZL006 could have a sustained effect simply by maintaining an effective concentration in vivo. It is plausible that treatment with ZL006 led to adaptive changes in neural circuits or alterations in gene expression, such as brain-derived neurotrophic factor (Cai et al. 2018a), which contributes to preventing aggressive behavior (Chang et al. 2020; Chang et al. 2018). Importantly, ZL006 ameliorated SI-induced escalation of attack behavior without disturbing the behavior in the GH mice, which is consistent with the finding of a previous study (Zhou et al. 2010). This specific effect of ZL006 on SI mice may attribute to aberrant NO level in SI mice but not in GH mice, further suggesting that ZL006 targets PSD95/ nNOS association and restores the deregulated NO level under pathological conditions (Cai et al. 2018b; Zhou et al. 2010). Therefore, ZL006 has an advantage over NMDAR and $\mathrm{nNOS}$ antagonists because it is designed to uncouple the NMDAR complex from nNOS in a targeted manner when a pathological insult occurs. It is worth noting that ZL006 probably blocks consolidation of contextual memory in aged mice (Aziz et al. 2019), suggesting that administration of ZL006 may have a detrimental effect in older individuals. Further work is required for delineation of the molecular and cellular mechanisms of ZL006 to understand the action of the drug and advance development of next-generation agents with fewer adverse effects.

Of note, we used smaller intruder animals of the same strain during the resident-intruder test. The main reason we chose 4-week-old mice as intruders is that mice in this age display fewer attack behaviors and would not have inflicted severe injuries during the test. Therefore, the exacerbated attack behavior of the resident mice subjected to SI may be regarded as abnormal or pathological (Miczek et al. 2001). It would be interesting to examine whether ZL006 could attenuate escalation of attack behavior when resident mice face intruder mice of similar size or those of a different strain in the future. We injected ZL006 via the i.p. or i.c.v. route in this study. Thus, a limitation of this research is that the specific brain region in which the drug acts remains to be determined. Nevertheless, our data show that systemic administration of ZL006 ameliorates SI-induced escalation of attack behaviors. We hope that our findings will prompt further studies to explore the neural circuits involved in attack behavior that are targeted by ZL006.

In conclusion, our study highlights the importance of the PSD95/nNOS pathway in the mediation of SI-induced escalation of attack behavior and suggests that targeting this pathway could be a promising therapeutic strategy for treating aggressive behaviors.

Supplementary Information The online version contains supplementary material available at https://doi.org/10.1007/s00213-021-06000-9.

Acknowledgements We would like to thank Dr. Heng Ai for critical reading of our manuscript. We also thank Editage (www.editage.cn) for English language editing.

Author contribution WL and LQY conceived and designed the study. WL and LQY performed the experiments and analyzed data. JYC and LGZ analyzed data. WL, JYC, LGZ, and LQY wrote the paper. All authors reviewed the results and approved the final version of the manuscript.

Funding This research has been funded by the National Natural Science Foundation of China Grant No. 31600863 (to Wen Lu).

\section{Declarations}

Conflict of interest The authors declare no competing interests.

\section{References}

Ai H, Shi XF, Hu XP, Fang WQ, Zhang B, Lu W (2017) Acute stress regulates phosphorylation of N-methyl-d-aspartate receptor GluN2B at S1284 in hippocampus. Neuroscience 351:24-35

Ai H, Fang W, Hu H, Hu X, Lu W (2020) Antidiabetic drug metformin ameliorates depressive-like behavior in mice with chronic restraint stress via activation of AMP-activated protein kinase. Aging Dis $11: 31-43$

Aziz W, Kraev I, Mizuno K, Kirby A, Fang T, Rupawala H, Kasbi K, Rothe S, Jozsa F, Rosenblum K, Stewart MG, Giese KP (2019) Multi-input synapses, but not LTP-strengthened synapses, correlate with hippocampal memory storage in aged mice. Curr Biol 29:3600-3610 e4

Baranyi A, Amouzadeh-Ghadikolai O, Rothenhausler HB, Theokas S, Robier C, Baranyi M, Koppitz M, Reicht G, Hlade P, Meinitzer A (2015) Nitric oxide-related biological pathways in patients with major depression. Plos One 10.

Brooks SK, Webster RK, Smith LE, Woodland L, Wessely S, Greenberg N, Rubin GJ (2020) The psychological impact of quarantine and how to reduce it: rapid review of the evidence. Lancet 395:912-920

Cacioppo S, Capitanio JP, Cacioppo JT (2014) Toward a neurology of loneliness. Psychol Bull 140:1464-1504

Cacioppo JT, Cacioppo S, Capitanio JP, Cole SW (2015) The neuroendocrinology of social isolation. Annu Rev Psychol 66:733-767

Cai CY, Chen C, Zhou Y, Han Z, Qin C, Cao B, Tao Y, Bian XL, Lin YH, Chang L, Wu HY, Luo CX, Zhu DY (2018a) PSD-95-nNOS 
coupling regulates contextual fear extinction in the dorsal CA3. Sci Rep-Uk 8.

Cai W, Wu S, Pan Z, Xiao J, Li F, Cao J, Zang W, Tao YX (2018b) Disrupting interaction of PSD-95 with nNOS attenuates hemorrhage-induced thalamic pain. Neuropharmacology 141:238-248

Carey LM, Lee WH, Gutierrez T, Kulkarni PM, Thakur GA, Lai YY, Hohmann AG (2017) Small molecule inhibitors of PSD95-nNOS protein-protein interactions suppress formalin-evoked Fos protein expression and nociceptive behavior in rats. Neuroscience 349:303-317

Carreno Gutierrez H, O'Leary A, Freudenberg F, Fedele G, Wilkinson R, Markham E, van Eeden F, Reif A, Norton WHJ (2020) Nitric oxide interacts with monoamine oxidase to modulate aggression and anxiety-like behaviour. Eur Neuropsychopharmacol 30:30-43

Chang CH, Su CL, Gean PW (2018) Mechanism underlying NMDA blockade-induced inhibition of aggression in post-weaning socially isolated mice. Neuropharmacology 143:95-105

Chang CH, Kuek EJW, Su CL, Gean PW (2020) MicroRNA-206 regulates stress-provoked aggressive behaviors in post-weaning social isolation mice. Mol Ther-Nucl Acids 20:812-822

Demas GE, Kriegsfeld LJ, Blackshaw S, Huang P, Gammie SC, Nelson RJ, Snyder SH (1999) Elimination of aggressive behavior in male mice lacking endothelial nitric oxide synthase. J Neurosci 19:RC30

Deyama S, Sugano Y, Mori S, Amano T, Yoshioka M, Kaneda K, Minami M (2017) Activation of the NMDA receptor-neuronal nitric oxide synthase pathway within the ventral bed nucleus of the stria terminalis mediates the negative affective component of pain. Neuropharmacology 118:59-68

Dhir A, Kulkarni SK (2011) Nitric oxide and major depression. Nitric Oxide 24:125-131

Doucet MV, Levine H, Dev KK, Harkin A (2013) Small-molecule inhibitors at the PSD-95/nNOS interface have antidepressant-like properties in mice. Neuropsychopharmacol 38:1575-1584

Eroglu E, Charoensin S, Bischof H, Ramadani J, Gottschalk B, Depaoli MR, Waldeck-Weiermair M, Graier WF, Malli R (2018) Genetic biosensors for imaging nitric oxide in single cells. Free Radical Bio Med 128:50-58

Filby AL, Paull GC, Hickmore TF, Tyler CR (2010) Unravelling the neurophysiological basis of aggression in a fish model. Bmc Genomics 11:498

Florio SK, Loh C, Huang SM, Iwamaye AE, Kitto KF, Fowler KW, Treiberg JA, Hayflick JS, Walker JM, Fairbanks CA, Lai Y (2009) Disruption of nNOS-PSD95 protein-protein interaction inhibits acute thermal hyperalgesia and chronic mechanical allodynia in rodents. Br J Pharmacol 158:494-506

Freudenberg F, Gutierrez HC, Post AM, Reif A, Norton WHJ (2016) Aggression in non-human vertebrates: genetic mechanisms and molecular pathways. Am J Med Genet B 171:603-640

Getting SJ, Segieth J, Ahmad S, Biggs CS, Whitton PS (1996) Biphasic modulation of GABA release by nitric oxide in the hippocampus of freely moving rats in vivo. Brain Research 717:196-199

Gunasekar PG, Kanthasamy AG, Borowitz JL, Isom GE (1995) NMDA receptor activation produces concurrent generation of nitric oxide and reactive oxygen species: implication for cell death. J Neurochem 65:2016-2021

Hevel JM, Marletta MA (1994) Nitric-oxide synthase assays. Methods Enzymol 233:250-258

Killgore WDS, Cloonan SA, Taylor EC, Anlap I, Dailey NS (2021) Increasing aggression during the COVID-19 lockdowns. J Affect Disord Rep 5:100163

Lee WH, Xu Z, Ashpole NM, Hudmon A, Kulkarni PM, Thakur GA, Lai YY, Hohmann AG (2015) Small molecule inhibitors of PSD95-nNOS protein-protein interactions as novel analgesics. Neuropharmacology 97:464-475
Lee WH, Carey LM, Li LL, Xu Z, Lai YY, Courtney MJ, Hohmann AG (2018) ZLc002, a putative small-molecule inhibitor of nNOS interaction with NOS1AP, suppresses inflammatory nociception and chemotherapy-induced neuropathic pain and synergizes with paclitaxel to reduce tumor cell viability. Mol Pain 14:1744806918801224

Li LP, Dustrude ET, Haulcomb MM, Abreu AR, Fitz SD, Johnson PL, Thakur GA, Molosh AI, Lai Y, Shekhar A (2018) PSD95 and nNOS interaction as a novel molecular target to modulate conditioned fear: relevance to PTSD. Transl Psychiatry 8:155

Miczek KA, Maxson SC, Fish EW, F Ac Cidomo S (2001) Aggressive behavioral phenotypes in mice. Behav Brain Res 125: 167-181.

Moncada S, Higgs A (1993) The L-arginine-nitric oxide pathway. N Engl J Med 329:2002-2012

Muir KW (2006) Glutamate-based therapeutic approaches: clinical trials with NMDA antagonists. Curr Opin Pharmacol 6:53-60

Mutlu O, Ulak G, Belzung C (2011) Effects of nitric oxide synthase inhibitors 1-(2-trifluoromethylphenyl)--imidazole (TRIM) and 7-nitroindazole (7-NI) on learning and memory in mice. Fundam Clin Pharmacol 25:368-377

Nelson RJ, Demas GE, Huang PL, Fishman MC, Dawson VL, Dawson TM, Snyder SH (1995) Behavioural abnormalities in male mice lacking neuronal nitric oxide synthase. Nature 378:383-386

Okruszek L, Aniszewska-Stanczuk A, Piejka A, Wisniewska M, Zurek K (2020) Safe but lonely? Loneliness, anxiety, and depression symptoms and COVID-19. Front Psychol 11:579181

O'Leary A, Laas K, Vaht M, Kiive E, Veidebaum T, Reif A, Harro J (2020) Nitric oxide synthase genotype interacts with stressful life events to increase aggression in male subjects in a populationrepresentative sample. Eur Neuropsychopharmacol 30:56-65

Olney JW, Labruyere J, Wang G, Wozniak DF, Price MT, Sesma MA (1991) NMDA antagonist neurotoxicity: mechanism and prevention. Science 254:1515-1518

Peterson JA, Chesbro G, Larson R, Larson D, Black CD (2021) Shortterm analysis ( 8 weeks) of social distancing and isolation on mental health and physical activity behavior during COVID-19. Front Psychol 12:652086

Pinna G, Dong E, Matsumoto K, Costa E, Guidotti A (2003) In socially isolated mice, the reversal of brain allopregnanolone down-regulation mediates the anti-aggressive action of fluoxetine. Proc Natl Acad Sci U S A 100:2035-2040

Qin C, Bian XL, Cai CY, Chen C, Zhou Y, Lin YH, Tao Y, Wu HY, Chang L, Luo CX, Zhu DY (2019) Uncoupling nNOS-PSD-95 in the ACC can inhibit contextual fear generalization. Biochem Biophys Res Commun 513:248-254

Qu W, Liu NK, Wu X, Wang Y, Xia Y, Sun Y, Lai Y, Li R, Shekhar A, Xu XM (2020) Disrupting nNOS-PSD95 interaction improves neurological and cognitive recoveries after traumatic brain injury. Cereb Cortex 30:3859-3871

Roy IL, Pothion S, Mortaud S, Habert CC, Nicolas L, Cherfouh A, Roubertoux PL (2000) Loss of aggression, after transfer onto a C57BL/6J background, in mice carrying a targeted disruption of the neuronal nitric oxide synthase gene. Behavior Genetics 30:367-373

Sattler R, Xiong Z, Lu WY, Hafner M, MacDonald JF, Tymianski M (1999) Specific coupling of NMDA receptor activation to nitric oxide neurotoxicity by PSD-95 protein. Science 284:1845-1848

Sherwin E, Lennon A, Harkin A (2017) Regional specific modulation of stress-induced neuronal activation associated with the PSD95/ NOS interaction inhibitor ZL006 in the Wistar Kyoto Rat. Int J Neuropsychopharmacol 20:833-843

Smith AE, Xu Z, Lai YY, Kulkarni PM, Thakur GA, Hohmann AG, Crystal JD (2016) Source memory in rats is impaired by an NMDA receptor antagonist but not by PSD95-nNOS proteinprotein interaction inhibitors. Behav Brain Res 305:23-29 
Tillmann S, Pereira VS, Liebenberg N, Christensen AK, Wegener G (2017) ZL006, a small molecule inhibitor of PSD-95/nNOS interaction, does not induce antidepressant-like effects in two genetically predisposed rat models of depression and control animals. Plos One 12:e0182698

Tripathi MK, Kartawy M, Amal H (2020) The role of nitric oxide in brain disorders: autism spectrum disorder and other psychiatric, neurological, and neurodegenerative disorders. Redox Biol 34

Wang ZY, Zhao Y, Jiang Y, Lv W, Wu L, Wang BY, Lv LY, Xu QW, Xin HL (2015) Enhanced anti-ischemic stroke of ZL006 by T-7-conjugated PEGylated liposomes drug delivery system. Sci Rep-Uk 5.

Zelikowsky M, Hui M, Karigo T, Choe A, Yang B, Blanco MR, Beadle K, Gradinaru V, Deverman BE, Anderson DJ (2018) The neuropeptide Tac 2 controls a distributed brain state induced by chronic social isolation stress. Cell 173:1265-+

Zhang J (2001) Nitric oxide synthase assays. Curr Protoc Pharmacol Chapter 2: Unit2 4.
Zhou Q, Sheng M (2013) NMDA receptors in nervous system diseases. Neuropharmacology 74:69-75

Zhou QG, Hu Y, Hua Y, Hu M, Luo CX, Han X, Zhu XJ, Wang B, Xu JS, Zhu DY (2007) Neuronal nitric oxide synthase contributes to chronic stress-induced depression by suppressing hippocampal neurogenesis. J Neurochem 103:1843-1854

Zhou L, Li F, Xu HB, Luo CX, Wu HY, Zhu MM, Lu W, Ji X, Zhou QG, Zhu DY (2010) Treatment of cerebral ischemia by disrupting ischemia-induced interaction of nNOS with PSD-95. Nat Med 16:1439-1443

Zhu LJ, Li TY, Luo CX, Jiang N, Chang L, Lin YH, Zhou HH, Chen C, Zhang Y, Lu W, Gao LY, Ma Y, Zhou QG, Hu Q, Hu XL, Zhang J, Wu HY, Zhu DY (2014) CAPON-nNOS coupling can serve as a target for developing new anxiolytics. Nat Med 20:1050-1054

Publisher's note Springer Nature remains neutral with regard to jurisdictional claims in published maps and institutional affiliations. 